The University of San Francisco

USF Scholarship: a digital repository @ Gleeson Library |

Geschke Center

1996

\title{
Oblique Electron Transport in the Presence of Collinear and Noncollinear Magnetizations
}

Horacio E. Camblong

University of San Francisco, camblong@usfca.edu

Follow this and additional works at: http://repository.usfca.edu/phys

Part of the Physics Commons

\section{Recommended Citation}

Camblong, Horacio E., "Oblique Electron Transport in the Presence of Collinear and Noncollinear Magnetizations" (1996). Physics and Astronomy. Paper 18.

http://repository.usfca.edu/phys/18 
AIP Applied Physics

Oblique electron transport in the presence of collinear and noncollinear magnetizations

Horacio E. Camblong

Citation: J. Appl. Phys. 79, 6383 (1996); doi: 10.1063/1.362006

View online: http://dx.doi.org/10.1063/1.362006

View Table of Contents: http://jap.aip.org/resource/1/JAPIAU/v79/i8

Published by the American Institute of Physics.

\section{Related Articles}

Towards compact three-dimensional magnetoelectronics-Magnetoresistance in rolled-up $\mathrm{Co} / \mathrm{Cu}$ nanomembranes

Appl. Phys. Lett. 100, 022409 (2012)

Large thermal Hall coefficient in bismuth

Appl. Phys. Lett. 100, 011903 (2012)

Magnon magnetoresistance of NiFe nanowires: Size dependence and domain wall detection

Appl. Phys. Lett. 99, 262504 (2011)

Large coercivity in nanostructured rare-earth-free MnxGa films

Appl. Phys. Lett. 99, 252506 (2011)

Large amplitude microwave emission and reduced nonlinear phase noise in $\mathrm{Co} 2 \mathrm{Fe}(\mathrm{Ge} 0.5 \mathrm{Ga} 0.5)$ Heusler alloy based pseudo spin valve nanopillars

Appl. Phys. Lett. 99, 162508 (2011)

\section{Additional information on J. Appl. Phys.}

Journal Homepage: http://jap.aip.org/

Journal Information: http://jap.aip.org/about/about_the_journal

Top downloads: http://jap.aip.org/features/most_downloaded

Information for Authors: http://jap.aip.org/authors

\section{ADVERTISEMENT}

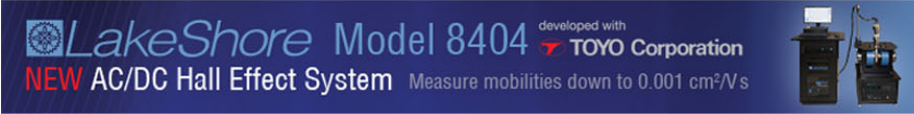




\title{
Oblique electron transport in the presence of collinear and noncollinear magnetizations
}

\author{
Horacio E. Camblong \\ Department of Physics, University of San Francisco, San Francisco, California 94117
}

The nonlocal real-space Kubo approach to electron transport in magnetic multilayers is applied to a new geometry, "oblique transport," whose complexity is traced back to a combination of the layering, of the nonlocal character of the linear response, and of the oblique direction transport in this new geometry. The problem is dealt with by applying a condition on the average current density vector or on the average electric-field vector, depending upon the external driving conditions. Its solution exhibits a characteristic anisotropy and it yields the global oblique conductance and magnetoresistance as simple trigonometric expressions in terms of the in-plane and vertical conductances and magnetoresistances, for arbitrary noncollinear-magnetization configurations. (C) 1996 American Institute of Physics.[S0021-8979(96)56308-8]

Electrical transport in metallic multilayers is fairly well understood and its applications to giant magnetoresistance have confirmed the validity of the various models that have been used, namely, the quasiclassical approach ${ }^{1}$ (based on the Boltzmann equation) and the Kubo approach. ${ }^{2-5}$

Previous theoretical analyses of magnetotransport in multilayers have only dealt with the special cases of in-plane transport (CIP) and vertical transport (CPP). Of course, there is a continuum of alternative geometries with the current neither in the plane of the layers nor perpendicular to the plane of the layers. Any such geometry is referred to as "oblique transport." The electric-field and current patterns for oblique transport are more involved than for CIP or CPP because variations in the local resistivity give rise to changes in the directions of the fields and currents from layer to layer. Actually, a version of this new geometry has been recently explored experimentally ${ }^{6}$ using a corrugated metallic multilayered structure. ${ }^{7}$

A typical oblique-transport scenario is shown in Fig. 1, where $z$ is a coordinate along the multilayer axis (or growth direction) and $\zeta$ is a coordinate along the "external driving direction," which I define as the direction of the average "driving field" $\mathbf{V}$. In practice, the driving field $\mathbf{V}$ will be either the current density $\mathbf{j}$ or the electric field $\mathbf{E}$; this leads to two outstanding boundary conditions: a "current-injection condition," when the direction of $\langle\mathbf{j}\rangle$ is externally fixed (for example, by proper insertion of current probes), and a "voltage condition," when the direction of $\langle\mathbf{E}\rangle$ is externally controlled. The parameter $\alpha$, which is defined as the angle between the axes $z$ and $\zeta$ (with $0^{\circ} \leqslant \alpha \leqslant 90^{\circ}$ ) measures the deviation of a particular oblique-transport geometry from either CIP $\left(\alpha=90^{\circ}\right)$, or CPP $\left(\alpha=0^{\circ}\right)$. These two coordinates are related via $\zeta=z \cos \alpha$, with a suitable choice of origin for both. Correspondingly, unit vectors along the external driving direction $\mathbf{e}_{\zeta}$, along the growth direction $\mathbf{e}_{z}$, and along the in-plane direction $\mathbf{e}_{x}$ satisfy the relations $\mathbf{e}_{\zeta} \cdot \mathbf{e}_{z}=\cos \alpha$ and $\mathbf{e}_{\zeta} \cdot \mathbf{e}_{x}=\sin \alpha$. In addition, it is clear that averages over $z$ and over $\zeta$ of $z$-dependent quantities are identical, i.e.,

$$
\langle f(z)\rangle_{z}=\langle f(\zeta / \cos \alpha)\rangle_{\zeta}
$$

As a consequence of the relations above, it follows that the component $A_{\zeta}(z)$ of any vector $\mathbf{A}(z)$ that is only $z$ dependent (for example, the current densities or the internal fields) has an average [cf. Eq. (1)]

$$
\left\langle A_{\zeta}(z)\right\rangle=\sin \alpha A_{\|}+\cos \alpha A_{\perp} .
$$

The analysis presented in this article is based on Eqs. (1) and (2), which are of interest when the relevant physical quantities (fields and currents) are essentially only $z$ dependent. Of course, this assumption breaks down near the external boundaries. Thus, for the computation of global properties, the analysis is valid when edge effects are globally unimportant.

The resulting transport behavior can be described most easily by using the real-space Kubo approach, ${ }^{3,4}$ wherein the general nonlocal linear relationship between the currents $\mathbf{j}_{\alpha \beta}(z)$ and the fields $\mathbf{E}_{\gamma \delta}\left(z^{\prime}\right)$ can be written as

$$
\mathbf{j}_{\alpha \beta}(z)=\int d z^{\prime} \sigma_{\alpha \beta, \gamma \delta}\left(z, z^{\prime}\right) \mathbf{E}_{\gamma \delta}\left(z^{\prime}\right),
$$

where the Greek indices label the two spin channels $(\alpha$ $=\uparrow, \downarrow), \sigma_{\alpha \beta, \gamma \delta}\left(z, z^{\prime}\right)$ is the spin-dependent two-point conductivity, and Einsteirix's sumpination convention for repeated indices has been appliec. In Eq. (3) the complex structure of

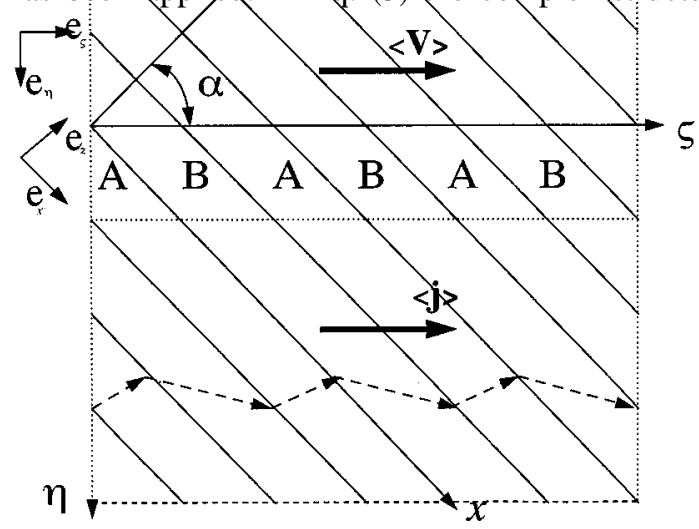

FIG. 1. Schematic representation of the oblique-transport geometry for a binary A/B superlattice grown along the $z$ direction. The dotted lines represent the bounds of a typical region; it is assumed that the system extends to infinity in both directions along the $\eta$ axis and that, if drawn to scale, many more layers would be encountered along the $\zeta$ axis. The lower part of the figure gives a graphical representation in terms of current lines of the current-injection condition (with $\sigma_{\mathrm{A}}<\sigma_{\mathrm{B}}$ ); the voltage-condition construction is not shown as it is more convoluted. 
spin indices is a convenient way of dealing with noncollinear magnetizations. In this framework the internal fields $\mathbf{E}_{\alpha \beta}(z)$ absorb the vertex corrections ${ }^{4}$ that correspond to long-range diffusion processes and acquire a spin dependence that can be thought of as arising from electrochemical potentials. $^{8,9}$

The geometries studied so far are simple in that either the current or the fields are essentially uniform: For CIP the external fields $\mathbf{E}_{\gamma \delta}(z)$ are constant (if a uniform external electric field is applied) and for CPP the current densities $\mathbf{j}_{\alpha \beta}(z)$ are constant. Instead, for oblique transport, both the currents and the fields are position dependent (even for a uniform external electric field) and their directions are not fixed in space. This complexity can be tackled by resolving all vector fields into components parallel $(\|)$ and perpendicular $(\perp)$ to the plane of the layers. This construction is useful due to the fact that geometrical symmetries associated with the multilayered system reduce both the spatial dependence and the vector structure of the two-point conductivity to a simple form. In effect, if edge effects are neglected, the twopoint conductivity is only a function of $z$ (twice) and that it admits the resolution into irreducible components: an inplane or CIP component, $\sigma_{\alpha \beta, \gamma \delta}^{(\|)}\left(z, z^{\prime}\right)$, and a vertical or CPP component, $\sigma_{\alpha \beta, \gamma \delta}^{(\perp)}\left(z, z^{\prime}\right)$. Explicitly,

$$
\sigma_{\alpha \beta, \gamma \delta}\left(z, z^{\prime}\right)=\mathbb{1}_{\|} \sigma_{\alpha \beta, \gamma \delta}^{(\|)}\left(z, z^{\prime}\right)+\mathbf{e}_{z} \mathbf{e}_{z} \sigma_{\alpha \beta, \gamma \delta}^{(\perp)}\left(z, z^{\prime}\right),
$$

where $\mathbb{1}_{\|}$is the unit tensor in the plane of the layers, and $\mathbf{e}_{z}$ is the unit vector in the $z$ direction. The approach of Refs. 3 and 4 yields the components of the two-point conductivity, $\sigma_{\alpha \beta, \gamma \delta}^{(r)}\left(z, z^{\prime}\right)$ (with $r=\|, \perp$ ), whose nonlocal spatial dependence is governed by the inverse length $\xi(z)$, that is proportional to $\Delta(z)$, the local scattering strength (imaginary part of the self-energy). Then, the resolution of the two-point conductivity into irreducible components, which is expressed by Eq. (4), leads to two independent problems. The CIP problem,

$$
j_{\alpha \beta}^{(\|)}(z)=\sigma_{\alpha \beta, \gamma \delta}^{(\|)}(z) E_{\gamma \delta}^{(\|)},
$$

has an apparent local character that is just an artifact generated by the uniformity of the externally applied electric field; in fact, Eq. (5) is formulated in terms of the one-point conductivity ${ }^{2,3} \sigma_{\alpha \beta, \gamma \delta}^{(\|)}(z)$, which is the integral of the twopoint conductivity with respect to $z^{\prime}$ and it governs the characteristic CIP exponential size effects (nonlocal effects), a result that is in agreement with experiments [see Eq.(8)]. On the other hand, the CPP problem,

$$
E_{\alpha \beta}^{(\perp)}(z)=\rho_{\alpha \gamma}(z) j_{\gamma \delta}^{(\perp)},
$$

is formulated in terms of the local resistivity ${ }^{4,9} \rho_{\alpha \beta}^{(\perp)}(z)$ $=C_{D}^{-1} \xi_{\alpha \beta}(z)$, which is a completely local quantity determined by the local scattering rate; this problem has the remarkable feature that the global conductance has no exponential size effects and is wholly determined by the so-called series resistor model or the average scattering, ${ }^{9-11}$ a prediction that has been found to be in impressive agreement with experimental results. ${ }^{12}$
In summary, the problems formulated by Eqs. (5) and (6) solve specifically in-plane and vertical transport. In addition, they can be used as the building blocks for oblique transport, as shown below.

For oblique transport it is necessary to consider the "matching" boundary conditions satisfied by the internal fields and the currents across the interfaces: namely, continuity of the tangential components of the fields $\mathbf{E}_{\alpha \beta}^{(\|)}$and continuity of the normal components of the currents $\mathbf{j}_{\alpha \beta}^{(\perp)}$. The condition on the internal fields follows from their irrotational character when defined in terms of the vertex corrections to the conductivity, ${ }^{4}$ whereas the condition on the currents expresses the independence of the spin channels, i.e., the twoindependent current model, an approximation that is valid when the spin-diffusion length is large (compared to the relevant scales for multilayers). ${ }^{4}$

Let us now investigate the variation in the directions of the fields and currents from layer to layer. The relationship between the fields and currents is indeed given by the nonlocal Eq. (3). However, from Eqs. (4)-(6), it follows explicitly that for a uniform externally applied electric field the corresponding internal fields are

$$
\mathbf{E}_{\alpha \beta}(z)=\mathbf{e}_{z} \rho_{\alpha \gamma}(z) j_{\gamma \beta}^{(\perp)}+\mathbf{e}_{\|} \rho_{\alpha \beta, \gamma \delta}^{(\|)}(z) j_{\gamma \delta}^{(\|)}(z),
$$

where $\rho_{\alpha \beta, \gamma \delta}^{(\|)}(z)$ is the matrix inverse with respect to $\gamma \delta$ of the one-point conductivity $\sigma_{\alpha \beta, \gamma \delta}(z)$. The interfacial boundary conditions further imply that $j^{(\perp)}$ and $E^{(\|)}$(second term) are constant. The two terms in Eq. (7) behave very differently because $\rho_{\alpha \gamma}(z)$ is a purely local quantity whereas $\rho_{\alpha \beta, \gamma \delta}^{(\|)}(z)$ has a nonlocal dependence through the local mean free paths. Let us see the limiting forms of $\rho_{\alpha \beta, \gamma \delta}^{(\|)}(z)$ : In the local limit (short mean free paths compared with layer thicknesses) it is asymptotically a tensor with components given by various rearrangements of indices of $\rho_{\alpha \gamma}(z)$, whereas in the homogeneous limit (long mean free paths compared with layer thicknesses) it is asymptotically given by $C_{D}^{-1} \bar{\xi}$. These different limiting behaviors indicate that, in the local limit, $\mathbf{E}$ is locally parallel to $\mathbf{j}$ but globally nonparallel, whereas in the homogeneous limit, $\mathbf{E}$ is globally parallel to $\mathbf{j}$ but locally nonparallel. In other words, the geometrical anisotropy associated with the layering prevents the currents and fields from being parallel to each other; thus, the medium behaves phenomenologically in an anisotropic way both locally and globally, a result that can be understood in terms of charge accumulation: Charges accumulate on the various interfaces to respect the various interfacial boundary conditions.

Averaging Eqs. (5) and (6) over the external-direction axis $\zeta$ gives

$$
\left\langle j^{(\|)}\right\rangle=\sigma_{\mathrm{CIP}} E^{(\|)}
$$

and

$$
\left\langle E^{(\perp)}\right\rangle=\rho_{\mathrm{CPP}} j^{(\perp)},
$$

where the averages are with respect to either $z$ or $\zeta$ [see Eq. (1)]. In Eq. (8)

$$
\sigma_{\mathrm{CIP}}=\left\langle\sigma_{\alpha \alpha, \gamma \gamma}^{(\perp)}(z)\right\rangle
$$

(with Einstein's summation convention for repeated indices) is the CIP global conductivity ${ }^{4}$ and in Eq. (9) 


$$
\rho_{\mathrm{CPP}}=\left(\sigma_{\mathrm{CPP}}\right)^{-1}=C_{D}^{-1}\left[\operatorname{Tr}\left(\bar{\xi}^{-1}\right)\right]^{-1}
$$

is the global CPP resistivity, with $\bar{\xi}=\langle\xi\rangle$ being proportional to the average scattering. ${ }^{4}$

The directly measurable quantities are the total voltage per unit length across the sample in the external driving direction,

$$
\frac{V}{L}=\frac{1}{L} \int \mathbf{E} \cdot d \mathbf{r}=\left\langle E_{\zeta}\right\rangle=\sin \alpha E^{(\|)}+\cos \alpha\left\langle E^{(\perp)}\right\rangle,
$$

[where $L$ is the length of the sample along the externaldriving direction, see Eq. (2)], and the average current density along the driving direction,

$$
\left\langle j_{\zeta}\right\rangle=\sin \alpha\left\langle j^{(\|)}\right\rangle+\cos \alpha j^{(\perp)} .
$$

In order to extract a global or measurable resistance from Eqs. (12) and (13), it is necessary to apply the external boundary conditions (see Fig. 1): either the current-injection condition, which is defined by

$$
\langle\mathbf{j}\rangle=\mathbf{e}_{\zeta}\left\langle j_{\zeta}\right\rangle
$$

or the voltage condition, which is defined by

$$
\langle\mathbf{E}\rangle=\mathbf{e}_{\zeta}\left\langle E_{\zeta}\right\rangle \text {. }
$$

Definition of the global resistivity as

$$
\rho=\frac{\left\langle E_{\zeta}\right\rangle}{\left\langle j_{\zeta}\right\rangle}
$$

leads to the following results:

$$
\rho_{1}=\rho_{\mathrm{CIP}} \sin ^{2} \alpha+\rho_{\mathrm{CPP}} \cos ^{2} \alpha,
$$

for current-injection conditions, and

$$
\sigma_{2}=\sigma_{\mathrm{CIP}} \sin ^{2} \alpha+\sigma_{\mathrm{CPP}} \cos ^{2} \alpha .
$$

for external-voltage conditions. Equations (17) and (18) are the main results of this article. They reduce to the familiar expressions $\rho\left(\alpha=90^{\circ}\right)=\rho_{\mathrm{CIP}}$ and $\rho\left(\alpha=0^{\circ}\right)=\rho_{\mathrm{CPP}}$, and they have the characteristic angular dependence of a quadratic form, i.e., they amount to the existence of a global resistivity tensor

$$
\boldsymbol{\rho}_{1}=1_{\|} \rho_{\mathrm{CIP}}+\mathbf{e}_{z} \mathbf{e}_{z} \rho_{\mathrm{CPP}}
$$

(such that $\rho=\mathbf{e}_{\zeta} \cdot \boldsymbol{\rho} \cdot \mathbf{e}_{\zeta}$ ) for current-injection conditions and to the existence of a global conductivity tensor

$$
\boldsymbol{\sigma}_{2}=1_{\|} \sigma_{\mathrm{CIP}}+\mathbf{e}_{z} \mathbf{e}_{z} \sigma_{\mathrm{CPP}}
$$

(such that $\sigma=\mathbf{e}_{\zeta} \cdot \boldsymbol{\sigma} \cdot \mathbf{e}_{\zeta}$ ) for external-voltage conditions.

The angular dependence of the magnetoresistance with respect to $\alpha$ can be derived straightforwardly from Eqs. (17) and (18). The main consequence stemming from this analysis for the resistivity and magnetoresistance is that the obliquetransport values are in between those for CIP and CPP, with the CPP values being the largest; this is because CPP is exactly self-averaging. ${ }^{4}$

In conclusion, I have calculated the oblique-transport electrical resistivity/conductivity and found that, as a consequence of the layering and if edge effects are unimportant, it is given as a quadratic form in the orientation of the externaldriving direction, with an anisotropic resistivity/conductivity tensor composed of the global CIP and CPP resistivities/ conductivities, for current-driving/external-voltage conditions. These results may be of help in the implementation of detailed phenomenological analyses of magnetotransport in magnetic multilayers.

\section{ACKNOWLEDGMENTS}

I would like to thank Professor P. M. Levy for providing a copy of his work prior to publication. This work was supported in part by the University of San Francisco Faculty Development Fund.

${ }^{1}$ R. E. Camley and J. Barnás, Phys. Rev. Lett. 63, 664 (1989); J. Barnás, A. Fuss, R. E. Camley, P. Grunberg and W. Zinn, Phys. Rev. B 42, 827 (1992).

${ }^{2}$ P. M. Levy, S. Zhang, and A. Fert, Phys. Rev. Lett. 65, 1643 (1990); S. Zhang, P. M. Levy, and A. Fert, Phys. Rev. B 45, 8689 (1992).

${ }^{3}$ H. E. Camblong and P. M. Levy, Phys. Rev. Lett. 69, 2835 (1992); J. Magn. Magn. Mater. 121, 446 (1993); H. E. Camblong, Phys. Rev. B 51, 1855 (1995).

${ }^{4}$ H. E. Camblong, P. M. Levy, and S. Zhang, Phys. Rev. B 51, 16052 (1995).

${ }^{5}$ A. Vedyayev, B. Dieny, and N. Ryzhanova, Europhys. Lett. 19, 329 (1992).

${ }^{6}$ T. Ono and T. Shinjo, J. Phys. Soc. Jpn. 64, 363 (1995).

${ }^{7}$ After completion of this article I have learned that a somewhat similar analysis of oblique transport is presented in P. M. Levy, S. Zhang, T. Ono, and T. Shinjo, Phys. Rev. B 52, 16049 (1995).

${ }^{8}$ P. M. Levy, H. E. Camblong, and S. Zhang, J. Appl. Phys. 75, 7076 (1994).

${ }^{9}$ H. E. Camblong, S. Zhang, and P. M. Levy, Phys. Rev. B. 47, 4735 (1993).

${ }^{10}$ S. Zhang and P. M. Levy, J. Appl. Phys. 69, 4786 (1991).

${ }^{11}$ H. E. Camblong and P. M. Levy, J. Appl. Phys. 73, 5533 (1993).

${ }^{12}$ W. P. Pratt, Jr., S.-F. Lee, J. M. Slaughter, R. Loloee, P. A. Schroeder, and J. Bass, Phys. Rev. Lett. 66, 3060 (1991); S.-F. Lee, W. P. Pratt, Jr., Loloee, P. A. Schroeder, and J. Bass, Phys. Rev. B 46, 548 (1992). 